\title{
SENSOR CALIBRATION OF THREE-LINE CCD SCANNERS ON ZY-3
}

\author{
Shenghui Fang, Yifu Chen \\ School of Remote Sensing and Information Engineering \\ Wuhan University, Wuhan 430079, China \\ shfang@whu.edu.cn, stone_syf@hotmail.com
}

Commission $\mathbf{I} / 3$

KEY WORDS: self-calibration, resolution, parameter, distortion, sensor

\begin{abstract}
:
ZY-3 is the first Chinese civilian high-resolution stereo mapping satellite. ZY-3 is equipped with three-line scanners (nadir, backward and forward views with $2.5 \mathrm{~m}$ and $4.0 \mathrm{~m}$ resolutions, respectively) and an additional multispectral scanner. In order to improve the mapping and positioning accuracy, sensor in-orbit calibration is a crucial procedure especially for high-resolution mapping-enabled satellites. The main focus of this paper is to describe the sensor calibration for ZY-3 sensors. Based on the experiences for other satellite sensor calibration, the following approach is proposed for ZY-3 sensor calibration in orbit: i) the initial internal geometric relationships of ZY-3 imaging sensors are established using the laboratory measurement and validation results; ii) assuming noise and errors are introduced once $\mathrm{ZY}-3$ is launched, a further investigation is conducted to detect those changed geometric relationships and their associated parameters, and detect and reduce highly correlated parameters. A self-calibration method is applied to identify and correct those parameters. Geometric calibration field data is used together with simulated ZY-3 data to perform the self-calibration; iii) the newly obtained parameters are used to establish the final geometric relationships model of ZY-3 imaging sensors, and with the aid of ground control points and check points from the geometric calibration field, those parameters are to be validated, and the position accuracy is also assessed by applying ZY-3 image data. Although promising results were obtained using ZY-3 simulated data, the proposed approach for ZY-3 imaging sensor calibration is an ongoing research work and needs to be fine tuned in the coming months.
\end{abstract}

\section{INTRODUCTION}

ZY-3 is the first Chinese civilian high-resolution stereo mapping satellite. ZY-3 is equipped with three-line scanners (nadir, backward and forward views with $2.5 \mathrm{~m}$ and $4.0 \mathrm{~m}$ resolutions, respectively) and an additional multispectral scanner. The resolutions of nadir, backward and forward views are $2.5 \mathrm{~m}, 4.0 \mathrm{~m}$ and $4.0 \mathrm{~m}$, respectively, and the resolution of the multispectral scanner is $8 \mathrm{~m}$. ZY-3 will be launched in early 2012 and orbit at 500-600km altitude sun-synchronously. The stereo base-height ratio is 0.85 0.95. The main purpose of ZY-3 data will be to map 1:50,000 cartographic maps, digital elevation model generation and generation of image ortho-rectification and map revision, and it will be used for many other applications such as notional resource surveying and environments monitoring.

Sensor calibration is a crucial procedure especially for high-resoluti mapping-enabled satellites such as SPOT, IKONOS, QuickBird, GeoEye, WorldView etc. The technical indicator of satellite's payload can be calibrated before the launch of the satellite in the laboratory. Before the remoter sensor manufacture has been completed, it is necessary to test and locate the interior orientation precisely and obtain the exact location of each pix at the image coordination systemso as to provide the basic geometry parameter information for the ground stereo-image surveying. After the injection of the satellite, it turns into micro-gravity status and the change in the environmental temperature status will cause the variation of the technical parameter of the satellite's payload (Jacobsen, 2003).

Laboratory calibration is not able to fully consider the condition of the image obtained by the satellite in the orbit, the error is inevitable. Therefore, it is necessary to conduct the in-orbit calibration for every technical parameter of the satellite's payload. Comprehensively analyze the each indicators of satellite running in the orbit which impact the mapping precision or the application in other industries, identify the reason which affect the application of satellite' data. Given that the satellite satisfies the design indicator, according to the result of calibration, we can adjust the rigorous imaging model and correct the factor that affects the geometric quality, in order to realize the high-precision position and mapping.

Performing the in-orbit geometric calibration for space borne sensor is very important and necessary for improving position accuracy and quality of image. On the one hand, the interior orientation elements, the geometric relationship among the CCD linear arrays and the placement's relationship among the platforms are able to be acquired according to in-orbit calibration. On the other hand, using the result of calibration is able to effectively eliminate the system error and the part of random error coming from the CCD linear arrays' distortion, shift and rotation and optics on lens' distortion etc (C.Valorge, 2003). in order to attain high geometric precision of imaging and improve position accuracy without GCP. Therefore, the in-orbit geometric calibration for satellite sensors is one of key technologies and essential parts to develop earth observation industry

\section{THREE CCD-LINES OF SATELLITE}

ZY-3 satellite is equipped with three high resolutions Pan-cameras, (nadir, backward and forward) and each Pan-camera has its own separate optical lens and CCD linear array independently. The resolutions of nadir, backward and forward views are $2.5 \mathrm{~m}, 4.0 \mathrm{~m}$ and $4.0 \mathrm{~m}$ respectively. In the along-track direction there is only a CCD sensor element; in the cross-track direction there are three CCD pieces which are spliced to a CCD linear array for acquiring a wide line image at an integral time. For the ZY-3 high-resolution satellite, the method of CCD line splicing technology adopts the optics splicing with a triple prism in order to get a higher splicing resolution. 


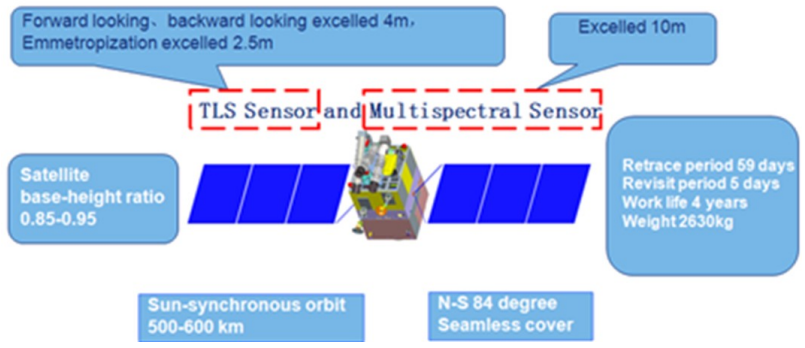

Figure 1: ZY-3 high-resolution stereo mapping satellite

\subsection{MULTI-LINE SENSORS IMAGING MODELS}

ZY-3 satellite is assembled with high-precision gyro, star sensor and dual-frequency GPS to acquire the high-precision satellite's attitude data and orbit data. In general, the acquired attitude data and orbit position data is belong to the different coordination system with perspective center of pan-camera, which there are a coordination displacement, so the data can't be used directly as satellite's exterior orientation. The orbit data presents the trace that the antenna of GPS runs in the space, but not the perspective center's orbit in the space (X. Tu, n.d.). In the self-calibration with ground control potions (GCP) using the rigorous imaging model, the displacement between the attitude and orbit position system and perspective center of pan-camera must be considered for constructing the sensor imaging model.
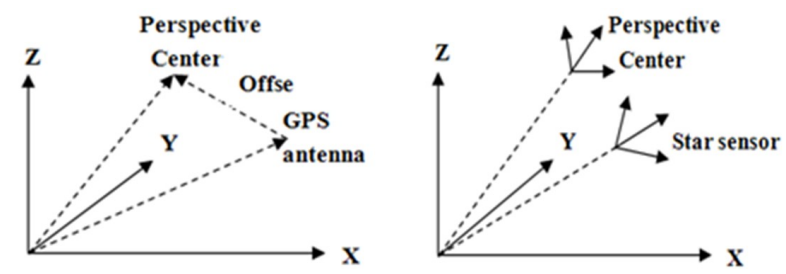

Figure 2: relationship among GPS antenna, Star sensor and Perspective center

During the procedure of the establishing a relationship between image and ground reference systems, the satellite body coordination system has been as the basic reference in order to consider the coordination displacements of the attitude and orbit position recording system. Using the laboratory calibration data of the displacements of GPS, star sensor and perspective center of pan-camera on the satellite' platform (satellite body coordination system), the relationship between the image space coordination system and the satellite body coordination system is presented by Equation.1.

$$
\left[\begin{array}{l}
x_{b} \\
y_{b} \\
z_{b}
\end{array}\right]=\left[\left[\begin{array}{l}
D_{x} \\
D_{y} \\
D_{z}
\end{array}\right]+\left[\begin{array}{l}
d_{x} \\
d_{y} \\
d_{z}
\end{array}\right]+R_{\text {camera }}^{\text {body }} \cdot\left(\begin{array}{l}
x \\
y \\
-f
\end{array}\right)\right]
$$

Where: $[x, y,-f]$ : point coordinates in the image system; $\left[x_{b}, y_{b}, z_{b}\right]:$ point coordinates in the satellite body system; $\left[d_{x}, d_{y}, d_{z}\right]$ : displacement between perspective center and satellite body system; [ $\left.D_{x}, D_{y}, D_{z}\right]$ : displacement between star sensor and satellite body system; $R_{\text {camera }}^{\text {body }}$ : rotation matrix from image space coordination system to satellite body system.

For the nadir pan-camera, the relationship from satellite body system to ground system also need, and rotation, $\mathrm{m}$ scale and trans- lation, which is described by Equation 2 .

$$
\begin{gathered}
{\left[\begin{array}{l}
X \\
Y \\
Z
\end{array}\right]_{W}=\left[\begin{array}{l}
X \\
Y \\
Z
\end{array}\right]_{C}+m \cdot R \cdot\left[\begin{array}{l}
x_{b} \\
y_{b} \\
z_{b}
\end{array}\right]} \\
R=R_{J 2000}^{W G S 4} \cdot R_{\text {orbit }}^{J 2000} \cdot R_{\text {body }}^{\text {orbit }}
\end{gathered}
$$

Where: $R_{\text {body }}^{\text {orbit }}$ : rotation matrix from satellite body system to satellite orbit system; $R_{\text {orbit }}^{J 2000}$ : rotation matrix from satellite orbit system to $\mathrm{J} 2000$ coordinate system; $R_{\text {orbit }}^{J 2000}$ : rotation matrix from J2000 coordination system to WGS84 coordinate system; $[X, Y, Z]_{C}^{T}$ : Perspective center position in WGS84 coordinate system; $[X, Y, Z]_{W}^{T}$ : Position coordinates in WGS84 coordinate system.

The equation 3 indicates the relationship between the image coordination system and ground coordination system WGS84 for the nadir pan-camera's perspective center.eq3

$$
\begin{aligned}
& {\left[\begin{array}{l}
X \\
Y \\
Z
\end{array}\right]_{W}=\left[\begin{array}{l}
X \\
Y \\
Z
\end{array}\right]_{C}+m \cdot R \cdot} \\
& \left.\left[\begin{array}{l}
D_{x} \\
D_{y} \\
D_{z}
\end{array}\right]+\left[\begin{array}{l}
d_{x} \\
d_{y} \\
d_{z}
\end{array}\right]+R_{\text {camera }}^{\text {body }} \cdot\left(\begin{array}{l}
x \\
y \\
-f
\end{array}\right)\right]
\end{aligned}
$$

For the forward and backward sensors, the relationship between image and ground reference systems is based on the nadir sensor by a rotation matrix (Poli, 2001). Forward sensor's imaging model is showed by equation 4 .

$$
\left[\begin{array}{l}
X \\
Y \\
Z
\end{array}\right]_{W}=\left[\begin{array}{l}
X \\
Y \\
Z
\end{array}\right]_{C}+m \cdot R \cdot R_{\text {Forward }} \cdot\left[\begin{array}{l}
x_{b} \\
y_{b} \\
z_{b}
\end{array}\right]
$$

Backward sensor's imaging model is showed by equation 5 .

$$
\left[\begin{array}{l}
X \\
Y \\
Z
\end{array}\right]_{W}=\left[\begin{array}{l}
X \\
Y \\
Z
\end{array}\right]_{C}+m \cdot R \cdot R_{\text {Backward }} \cdot\left[\begin{array}{l}
x_{b} \\
y_{b} \\
z_{b}
\end{array}\right]
$$

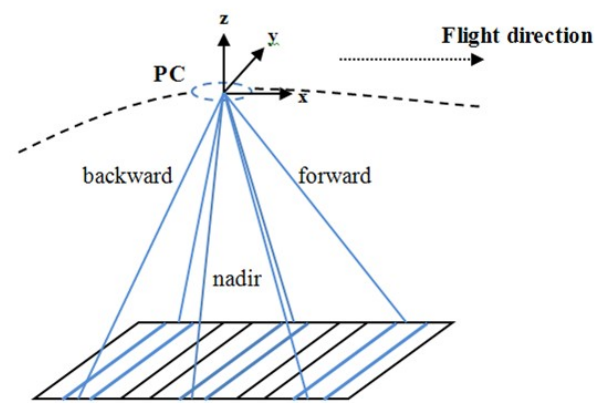

Figure 3: Three CCD linear array sensors (forward, nadir, backward sensor)

When satellite's manufacturing and assembly is complete, it need to be calibrated in laboratory, so that the offsets $D_{x}, D_{y}, D_{z}, d_{x}$, $d_{y}, d_{z}$ and the rotation matrix can be as a fix value ,equation 6. The relationship between image and ground reference systems, equation 3 , is simple to equation 7 .

$R_{C}=m \cdot R_{J 2000}^{W G S 84} \cdot R_{\text {orbit }}^{J 2000} \cdot R_{\text {body }}^{\text {orbit }} \cdot\left[\left[\begin{array}{c}D_{x} \\ D_{y} \\ D_{z}\end{array}\right]+\left[\begin{array}{c}d_{x} \\ d_{y} \\ d_{z}\end{array}\right]\right]$ 


$$
\left[\begin{array}{l}
X \\
Y \\
Z
\end{array}\right]_{W}=\left[\begin{array}{c}
X \\
Y \\
Z
\end{array}\right]_{C}+m \cdot R \cdot R_{\text {camera }}^{\text {body }} \cdot\left[\begin{array}{c}
x \\
y \\
-f
\end{array}\right]+R_{C}
$$

\subsection{RECONSTRUCTING GEOMETRIC RELATIONSHIP MODEL}

When satellite is running in its orbit, the environmental condition is different from the ground, such as micro-gravity, temperature and humidity etc, therefore the technical parameters of the highresolution camera on the satellite and the collinear form established through image point, perspective center and ground point will change. It makes the position of image point on the focal plan deviate from theory position calibrated in the laboratory, thus to cause a system error. In order to realize the high-precision inorbit calibration for CCD linear array sensor, it is very necessary and important to build the reasonable error model basing on the analysis of satellite's structural parameters. In this study, the system error coming from the interior orientation, radial direction and tangential direction distortion of optics lens and CCD-line's distortion and rotation will be modeled mainly.

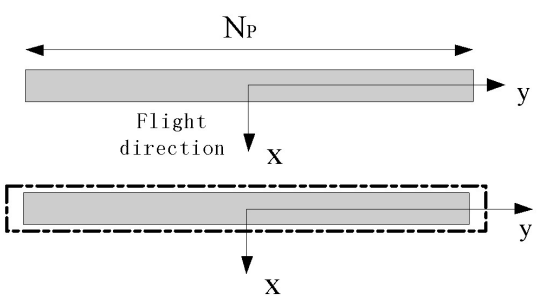

Figure 4: Expansive and contractive distortions of CCD linear array

2.2.1 CCD pixel distortion Due to the temperature change's factor, the CCD pixel in the camera could be make expansive and contractive distortions. Each CCD pixel distortion in $\mathrm{x}$ and $\mathrm{y}$ direction are presented by and. In the $\mathrm{x}$ direction, there is just one pixel, so that the distortion of pixel is every small. But in the cross orbit direction, there are a large number of CCD pixels that construct a CCD-line, so that the distortion can't be ignored that presented by .

$$
\left\{\begin{array}{l}
d p_{x}=d x_{p} \\
d p_{y}=N_{p} \cdot d y_{p} \quad d p_{y}=s y
\end{array}\right.
$$

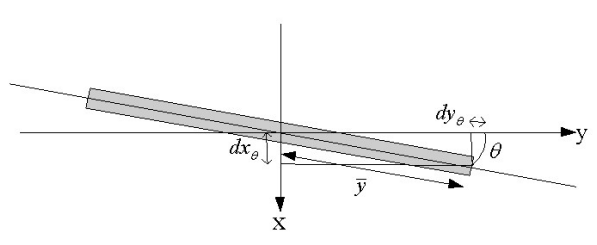

Figure 5: CCD-line rotation

2.2.2 CCD-line rotation During the procedure of satellite launching, the satellite will get various vibration, thus to make a CCD -line rotate a micro angle against the true installation sit. The rotation angle is presented by theta in the figure 5 . Because of the rotation angle theta is very small, $d y_{\theta}$ is able to be ignored, $d y_{\theta} \approx 0$, thus only to consider the distortion .

$$
\left\{\begin{array} { l } 
{ d x _ { \theta } = \overline { y } \operatorname { s i n } \theta } \\
{ d y _ { \theta } = \overline { y } - \overline { y } \operatorname { c o s } \theta = \overline { y } ( 1 - \operatorname { c o s } \theta ) }
\end{array} \quad \left\{\begin{array}{l}
\bar{x}=x-x_{0} \\
\bar{y}=y-y_{0}
\end{array}\right.\right.
$$
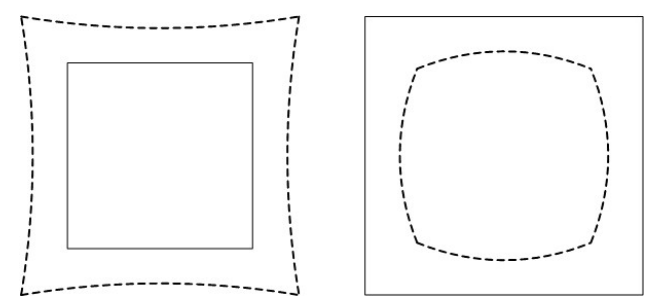

Figure 6: Radial direction and tangential direction distortion of optics lens

2.2.3 Radial direction and tangential direction distortion of optics lens Lens optical distortion is caused by design, production, and assembly of lens optical system, resulting in image point deviating from the idea position. Usually the lens distortion consists of radial and tangential distortions.

Radial direction distortion

The lens optical distortion is modeled with odd polynomial, showed by equation 10 . In order to obtain the distortions in $\mathrm{x}$ direction and y direction, the distortion is decomposed into $\Delta x_{r}$ and $\Delta y_{r}$ , presented by equation 11 .

$$
\begin{gathered}
d r=k_{1} r^{3}+k_{2} r^{5}+k_{3} r^{7}+\cdots \\
\left\{\begin{array}{c}
\Delta x_{r}=k_{1} \bar{x} r^{2}+k_{2} \bar{x} r^{4}+k_{3} \bar{x} r^{6}+\ldots \\
\Delta y_{r}=k_{1} \bar{y} r^{2}+k_{2} \bar{y} r^{4}+k_{3} \bar{y} r^{6}+\ldots \\
\bar{x}=\left(x-x_{0}\right) \\
\bar{y}=\left(y-y_{0}\right) \\
r=\sqrt{x^{2}+y^{2}}
\end{array}\right.
\end{gathered}
$$

where $\left(x_{0}, y_{0}\right)$ : Principal point of photograph

2.2.4 Tangential direction distortion The tangential distortion is caused mainly by the optical center of optical system disagreeing with geometric center of optical system. In the other word, the optical center of lens is not rigorous collinear, which is showed in figure 7 and modeled by equation 12 in $\mathrm{x}$ and $\mathrm{y}$ directions.
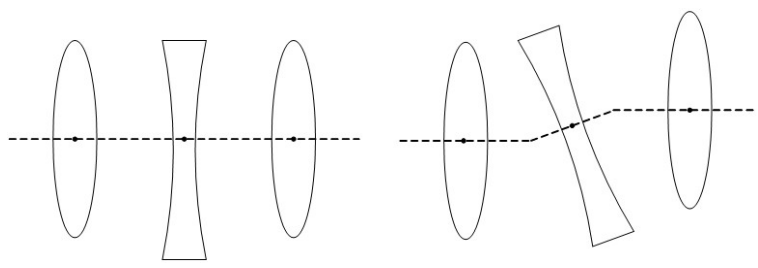

Figure 7: the optical center of lens is not rigorous collinear

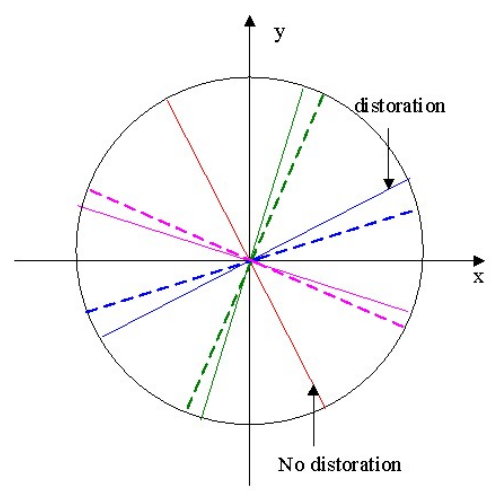

Figure 8: Tangential distortion 


$$
\left\{\begin{array}{c}
\Delta x_{d}=P_{1}\left(r^{2}+2 \bar{x}^{2}\right)+2 P_{2} \bar{x} \cdot \bar{y} \\
\Delta y_{d}=P_{2}\left(r^{2}+2 \bar{y}^{2}\right)+2 P_{1} \bar{x} \cdot \bar{y} \\
P(r)=\sqrt{P_{1}^{2}+P_{2}^{2}} \cdot r^{2}
\end{array}\right.
$$

Based on the above impact factors, the total errors of image point in $\mathrm{x}$ and $\mathrm{y}$ direction $\mathrm{s}$ is indicated by equation 13, interior orientation model.

$\left\{\begin{array}{l}\Delta x=x_{0}+\left(k_{1} r^{2}+k_{2} r^{4}\right) \bar{x}+p_{1}\left(r^{2}+2 \bar{x}^{2}\right)+2 p_{2} \bar{x} \bar{y}+\bar{y} \sin \theta \\ \Delta y=y_{0}+\left(k_{1} r^{2}+k_{2} r^{4}\right) \bar{y}+p_{2}\left(r^{2}+2 \bar{y}^{2}\right)+2 p_{1} \bar{x} \bar{y}+s_{y} \bar{y}\end{array}\right.$

In additional to the above factors, interior orientation, the satellite's orbit and attitude errors are important errors original for geometric accuracy of satellite's imaging. Therefore, introducing the rotation error matrix $R_{\text {error }}$ is necessary for eliminating the system errors and a part of random error from attitude, a part of residual error from interior orientation corrected and some unknown error. $R_{\text {error }}$ is a complex compounded errors model that is presented by equation 14 .

$$
\begin{gathered}
R_{\text {error }}=\left[\begin{array}{ccc}
K 1 & K 2 & K 3 \\
P 1 & P 2 & P 3 \\
Q 1 & Q 2 & Q 3
\end{array}\right] \\
{\left[\begin{array}{c}
X \\
Y \\
Z
\end{array}\right]_{W}=\left[\begin{array}{c}
X \\
Y \\
Z
\end{array}\right]_{C}+m \cdot R \cdot R_{\text {error }} \cdot R_{\text {camera }}^{\text {body }} \cdot\left[\begin{array}{c}
x+\Delta x \\
y+\Delta y \\
-f
\end{array}\right]}
\end{gathered}
$$

The equation 7, 13 and 14 are combined to the equation 15 for inorbit self-calibration. The parameters of self-calibration include total 18 parameters as $x_{0}, y_{0}, f, K 1, K 2, K 3, P 1, P 2, P 3, Q 1$, $Q 2, Q 3, k 1, k 2, p 1, p 2, \theta, s y$.

\section{SELF-CALIBRATION'S SOLUTION MODEL}

During the procedure of solution with additional self-calibration parameters, weight value of each kind of observation value is introduce into the least square adjustment through building virtual observation function for each kind of observation value (Poli, n.d.). In this study, the image point, GCP, c1 parameter and c2 parameter are observations so that every kind of weight value of observation value can be introduced in order to improve the accuracy of solution and the adjustment error model is presented by equation 16.

$$
\left\{\begin{array}{lrrrr}
V_{I}=A x+B t+C_{1} c 1+C_{2} \cdot c 2 & -L_{I} & & p_{I} \\
V_{G}=E_{x} x & & & -L_{G} & p_{G} \\
V_{C 1}= & E_{c 1} c 1 & & -L_{C 1} & p_{C 1} \\
V_{C 2}= & & E_{c 2} c 2 & -L_{C 2} & p_{C 2}
\end{array}\right.
$$

Where: $V_{I}, V_{G}, V_{C 1}, V_{C 2}$ :vector of observational correction of image point coordination, ground control point coordination, self-calibration parameter and error rotational matrix. $x$ : vector of GCP'S correction; $t$ :vector of exterior orientation correction. $c 1$ :vector of self-calibration parameter $c 2$ :vector of error rotational matrix ; $A, B, C_{1}, C_{2}$ :coefficient matrix of $x, t, c 1, c 2$ in bundle block adjustment; $L_{I}, L_{G}, L_{C 1}, L_{C 2}$ :residual vector of observation value; $P_{I}, P_{G}, P_{C 1}, P_{C 2}$ :weight value of observation value;

\section{SIMULATION DATA ACQUSITION AND PREPARATION}

Simulation data of ZY-3 satellite is acquired by using the laboratorycalibration's data, orbit and attitude value of satellite and high resolution aerial photogrammetry. According to the high-accurate image matching the image coordination and objective coordination of points are attained from aerial photogrammetry as ground $\theta$ control points used in self-calibration GCP. The procedure of simulating data: i) the regular distributed GCP is simulated in imaging region covered by satellite. ii) using designed parameter file, data from laboratory calibration and validation results simulates exterior orientation elements; iii) according to the regular distributed GCP and simulated exterior orientation elements, the image point without error is simulated by co-linearity equation; iiii) check the correctness of the satellite simulated data. Using stereo image calculates ground point with forward intersection. If the coordinate of the calculated ground point is equal to the simulated GCP, the simulate data is correct.

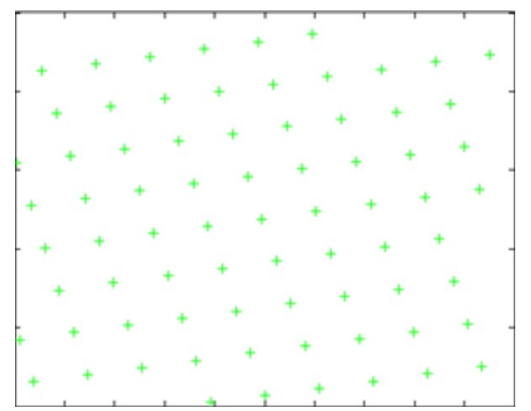

Figure 9: 74 GCPs of self-calibration

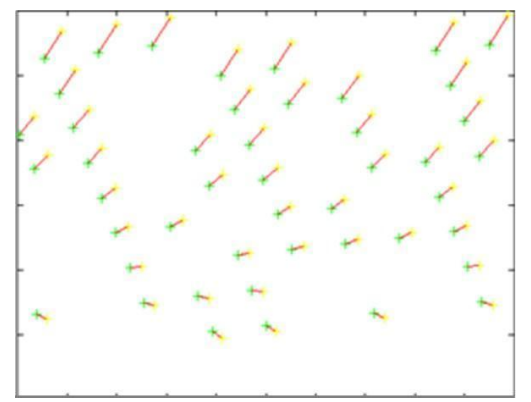

Figure 10: Image point of GCP with errors

In order to study and test the correctness of the method of selfcalibration described in this study, error is introduced into image point, standard deviation in $\mathrm{x}$ direction 0.4 , in y direction 0.6 . In our simulated data, there are 74 ground control points (GCP), 25 of which are used for check points. In figure 9, the green cross presents ground control point distributed regularly, and the red line presents introduced errors showed in figure 10.

\section{CALIBRATION AND RESULT}

i) According to ground control point, using bundle block adjustment solutes self-calibration parameter $\mathrm{c} 1$ and error rotation matrix c2; ii) Using 27 check points check and analyze the result of calibration; iii) Analyzing the accuracy of the result to evaluate the self-calibration method's correctness; iii) using the different number of GCP acquires calibration's results and compares the different accuracies to analyze the influence of the number of GCP on the accuracy of calibration. iiii) the reasonable parameter$s$ of self-calibration are determined by analyzing the covariance 
matrix of bundle block adjustment; iiiii) the new self-calibration parameters are used to calibration's calculation for determining the correctness and stability of the new selected self-calibration's parameter in the condition of using different number of GCP.

In the procedure of solution, there are some correlations generated between the parameter $\mathrm{c} 1\left(x_{0}, y_{0}, f, k_{1}, k_{2}, p 1, p 2, \theta, s y\right)$ and c2(K1,K2K3,P1, $22, P 3, Q 1, Q 2, Q 3)$. Meanwhile, c1 has a correlation among itself. In order to eliminate the influence of the correlation between $\mathrm{c} 1$ and $\mathrm{c} 2$, the iterative solution of $\mathrm{c} 1$ and $\mathrm{c} 2$ are separated. Firstly, the parameter $\mathrm{c} 1$ is calculated iteratively with initial parameter's value, and then the parameter $\mathrm{c} 2$ is calculated iteratively using the value of parameter $\mathrm{c} 1$. Finally, the self-calibration value of parameter $\mathrm{c} 1$ and $\mathrm{c} 2$ are determined by reiterating several times interactions.

Parameter c2 includes a few of errors such as residual deviation from c1, system deviation of exterior orientation elements and some random errors, therefore it is very difficult to separate out each kind of errors respectively. The errors rotation matrix can only be used to improve the positioning accuracy and surveying accuracy.

\subsection{Self-calibration parameters}

According to comparing the calculated result of parameter $\mathrm{c} 1 \mathrm{us}$ ing the different number of ground control points $(46,36,26$ and $16 \mathrm{GCP})$, the value of parameter $Y_{0}, K_{1}, K_{2}, s y$ is steady, but the value of parameter $x_{0}, f, p_{1}, p_{2}$ is vibrated in different number of GCP. The correlation among parameter $x_{0}, y_{0}, f, p 1, p 2, \theta$ is high during the analyzing the covariance matrix. According to analysis and experiment, the new reasonable self-calibration parameters are determined as $x_{0}, y_{0}, f, k_{1}, k_{2}, \theta, s y$.

Table 1: The assessment using check points for 18 parameters.

\begin{tabular}{lllll}
\hline \multicolumn{5}{l}{$K_{1}, K_{2}, K_{3}, P_{1}, P_{2}, P_{3}, Q_{1}, Q_{2}, Q_{3}}$, \\
$x_{0}, y_{0}, f, k_{1}, k_{2}, p 1, p 2$, Theta, sy & (18) \\
\hline Num GCP & $P$ & $\sigma_{x}$ & $\sigma_{v}$ & $\sigma_{\text {sum }}$ \\
\hline 46 & 0.038 & 0.0159 & 0.0787 & 0.08029 \\
\hline 36 & 0.030 & 0.0158 & 0.0783 & 0.07984 \\
\hline 26 & 0.025 & 0.0158 & 0.0548 & 0.05706 \\
\hline 16 & 0.100 & 0.0134 & 0.0740 & 0.07524 \\
\hline
\end{tabular}

Table 1presents the different assessment's results using check points (CP) and the value of self-calibration's parameters calculated with the various number of ground control points (GCP) for $18 \mathrm{cal}-$ ibration's parameters. The results are given in terms of standard deviation values (sigma). The check points a subset of the 74GCPs, which are not used as control points for the adjustmen$\mathrm{t}$ (Kocaman, 2003). The sigma values vary between 0.080290.05706 pixel. The min sigma value in the $\mathrm{x}$ and $\mathrm{y}$ directions are 0.0134 and 0.0548 pixel, corresponding for the 16 and 26 GCPs respectively.

\subsection{Self-calibration parameters}

Analyzing the value of new determined self-calibration's parameter calculated in different number of GCP $(46,36,26$ and 16 GCP), the parameter $x_{0}, y_{0}, k_{2}, \theta$ is not steady yet. According to analysis, the reason is that the parameter $\theta$ has a certain correlation with principle point $x_{0}, y_{0}$ yet, thus the parameter $\theta$ is removed and the new self-calibration parameters are determined as $x_{0}, y_{0}, k_{1}, k_{2}$, sy again.

Table 2 presents the different assessment's results using check points (CP) and the value of self-calibration's parameters calculated with the various number of ground control points (GCP) for
Table 2: The assessment using check points for 15 parameters.

\begin{tabular}{lcccc}
\hline \multicolumn{4}{l}{$K_{1}, K_{2}, K_{3}, P_{1}, P_{2}, P_{3}, Q_{1}, Q_{2}, Q_{3}$} \\
\multicolumn{1}{l}{$x_{0}, y_{0}, k_{1}, k_{2}$, Theta, sy } \\
\hline Num GCP & $P$ & $\sigma_{x}$ & $\sigma_{v}$ & $\sigma_{\text {sum }}$ \\
\hline 46 & 0.077 & 0.0215 & 0.0767 & 0.07966 \\
\hline 36 & 0.001 & 0.0029 & 0.0802 & 0.08028 \\
\hline 26 & 0.100 & 0.0533 & 0.0146 & 0.05529 \\
\hline 16 & 0.061 & 0.0163 & 0.0752 & 0.07700 \\
\hline
\end{tabular}

15 calibration's parameters. The sigma values are assessed with $2 \mathrm{t}$ check points and vary between $0.5529-0.08028$ pixel. When the number of GCP is 26, the standard deviation is minimum, 0.5529 pixel.

\subsection{Self-calibration parameters}

For the 14 self-calibration parameters, the results are calculated with 46, 36, 26 and 16GCPs respectively. It is obvious that the correlation among parameter $\mathrm{c} 1$ almost is estimated according to analyze covariance matrix. The value of calibration's parameter $\mathrm{c} 1$ is steady relatively for using the different number of GCP.

Table 3: The assessment using check points for 14 parameters.

\begin{tabular}{ccccc}
\hline$K_{1}, K_{2}, K_{3}, P_{1}, P_{2}, P_{3}, Q_{1}$, & & \\
$Q_{2}, Q_{3}, x_{0}$, & $y_{0}, k_{1}, k_{2}, s y$ & & $(14)$ \\
\hline Num GCP & $P$ & $\sigma_{x}$ & $\sigma_{v}$ & $\sigma_{\text {sum }}$ \\
\hline 46 & 0.077 & 0.0215 & 0.07674 & 0.07931 \\
\hline 36 & 0.001 & 0.0217 & 0.0706 & 0.07396 \\
\hline 26 & 0.100 & 0.0146 & 0.0508 & 0.05293 \\
\hline 16 & 0.060 & 0.0161 & 0.0725 & 0.07431 \\
\hline
\end{tabular}

The assessment results are given in table 3. The standard devastation values are lower than the other tests and vary between 0.05293-0.07931. The minimum is equal to 0.05293 pixel $\mathrm{t}$ calculating with 26 GCPs.

\subsection{Result's analysis}

Comparing with table 1, 2 and 3, it is obvious that the standard deviations calculated with the result of calibration using $26 \mathrm{GCP}$ $\mathrm{s}$ are $0.05706,0.05529$ and 0.05293 with the different forms of self-calibration's parameters. The values are minimum respectively in the conditions using the different number of GCPs and self-calibration's parameters, which means the result of calibration will be influenced by the number and distribution of GCP. According to comparing table1,2, and 3 , we can find the values of standard deviation in table 3 using 16, 26, 36, and 46 GCPs are totally lower than the values in table 1 and table 2 . The comparing result indicates the accuracy of correction for check point will increase using the same GCPs when the correlation among self-calibration's parameters is eliminated to some extent. Figure $11 \mathrm{~b}$ is the result of correction with $\mathrm{c} 1\left(x_{0}, Y_{0}, K_{1}, K_{2}, s y\right)$, c2 ( $K_{1}, K_{2}, K_{3}, P_{1}, P_{2}, P_{3}, Q_{1}, Q_{2}, Q_{3}$ ) self-calibration's paradises and 26 GCPs. According to comparing the figure 11 (a) and (b), it is obvious that the system errors showed in (a) are eliminated nearly, thus to prove the correctness of the in-orbit calibration method proposed in this paper.

\section{CONCLUSIONS}

In this study, the geometric relationships of ZY-3 from image system to ground system are established using the laboratory mea- 


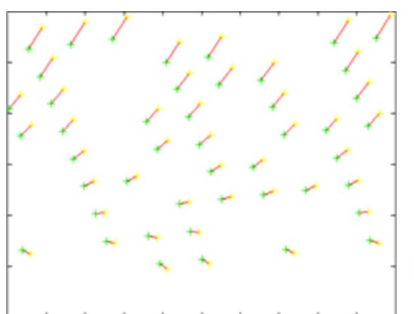

(a)

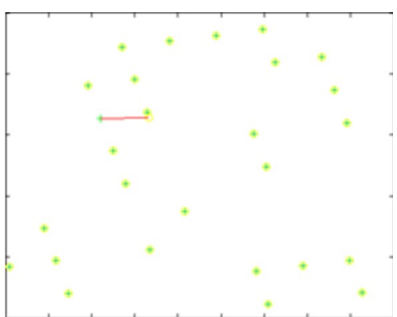

(b)
Figure 11: Result of correction with $c 1\left(x_{0}, y_{0}, k_{1}, k_{2}, s y\right)$ and $c 2$ self-calibration's paradises for CP. (a): GCP with errors (b):CP corrected by the result of calibration

surement and validation results. According to studying and analyzing ZY-3 sensor model and self -calibration adjustment mod$\mathrm{el}$, the geometric calibration method is proposed for three CCD linear array sensors. The calibration method presented in the paper was verified using ZY-3 simulated data and the result of experiment showed its correctness and feasibility for ZY-3 PANcamera's in-orbit calibration. Because of the study' main aim is to confirm the correctness and feasibility of the calibration method, the experiment is only done for single image. Therefore, as future work, the current in-orbit calibration method will be further investigated and tested using multi-image from different sensors in different time and which distribution of GCP can acquire higher calibration's accuracy with this calibration's method. Moreover the trajectory modeling functions will be studied for ZY-3.The true feasibility and accuracies need to be verified using the actual ZY-3 data once it is launched. It is important and necessary to explore the potentials of using ZY-3 data to map regions without ground control information such as the western part of China where accessibility is limited due to high altitude mountains.

\section{REFERENCES}

C.Valorge, 2003. 40 years of experience withspot in-flight calibration. Workshop on Radiometric and Geometric Calibration, Gulfport.

Jacobsen, K., 2003. Issues and method for in-flight and on-orbit calibration. Workshop on Radiometric and Geometric Calibration.

Kocaman, S., 2003. Self-calibrating triangulation with tls imagery. Internal Technical Report.

Poli, D., 2001. Direct georeferencing of multi-line images with a general sensor model. ISPRS Workshop.

Poli, D., n.d. General model for airborne and spaceornelinear array sensors. Proceedings of ISPRS Commission I Symposium.

X. Tu, M. Xu, L. L., n.d. The geometric calibration of airborne three-line scanner ads40. Acta Geoda etica et Ca rtographica Sinica. 